\title{
Comparison of simplified theories in the analysis of the diffraction efficiency in surface-relief gratings
}

\author{
J. Francés ${ }^{\mathrm{a}, \mathrm{b}^{*}}$, C. Neipp ${ }^{\mathrm{a}, \mathrm{b}}$, S. Gallego ${ }^{\mathrm{a}, \mathrm{b}}$, S. Bleda ${ }^{\mathrm{a}, \mathrm{b}}$, A. Márquez ${ }^{\mathrm{a}, \mathrm{b}}$, I. Pascual ${ }^{\mathrm{b}, \mathrm{c}}$, A. Beléndez $^{\mathrm{a}, \mathrm{b}}$ \\ ${ }^{a}$ Dept. of Physics, Systems Engineering and Signal Theory, Alicante Univ./San Vicente del Raspeig \\ Drive, San Vicente del Raspeig, Alicante, España E-03080 \\ ${ }^{b}$ University Institute of Physics to Sciencies and Technologies, Alicante Univ./ San Vicente del \\ Raspeig Drive, San Vicente del Raspeig, Alicante, España E-03080 \\ ${ }^{c}$ Dept. of Optics, Pharmacology and Anatomy, Alicante Univ./ San Vicente del Raspeig Drive, San \\ Vicente del Raspeig, Alicante, España E-03080
}

\begin{abstract}
In this work a set of simplified theories for predicting diffraction efficiencies of diffraction phase and triangular gratings are considered. The simplified theories applied are the scalar diffraction and the effective medium theories. These theories are used in a wide range of the value $\Lambda / \lambda$ and for different angles of incidence. However, when $1 \leq \Lambda / \lambda \leq 10$, the behaviour of the diffraction light is difficult to understand intuitively and the simplified theories are not accurate. The accuracy of these formalisms is compared with both rigorous coupled wave theory and the finite-difference time domain method. Regarding the RCWT, the influence of the number of harmonics considered in the Fourier basis in the accuracy of the model is analyzed for different surface-relief gratings. In all cases the FDTD method is used for validating the results of the rest of theories. The FDTD method permits to visualize the interaction between the electromagnetic fields within the whole structure providing reliable information in real time. The drawbacks related with the spatial and time resolution of the finite-difference methods has been avoided by means of massive parallel implementation based on graphics processing units. Furthermore, analysis of the performance of the parallel method is shown obtaining a severe improvement respect to the classical version of the FDTD method.
\end{abstract}

Keywords: Diffraction gratings, RCWA, FDTD, Scalar diffraction theory, EFM

\section{INTRODUCTION}

In this paper, we show the results obtained by a set of several theories that predict the diffraction properties of two different optical components, such as binary phase gratings and triangular gratings. Specifically, the gratings have been studied in the resonance domain having periods ranging from one to ten wavelengths. Binary phase gratings are of wide interest owing to many applications in quantum electronics, integrated optics, spectroscopy, and holography ${ }^{1-2}$. Triangular gratings are promising for applications requiring antireflection, polarization selection and spreading, which are best explained in terms of diffractive optics rather than ray optics ${ }^{4}$.

Different rigorous electromagnetic vector theories are frequently applied to yield exact diffraction performances. The accuracy of these theories is insensitive to the feature size of the Diffractive Optical Elements (DOE). However, the rigorous vector methods are more complicated than some simplified theories and usually are computationally intensive.

The Scalar Diffraction Theory (SDT) and the Effective Medium Theory (EMT) are considered as a good alternative for predicting diffraction efficiency of DOEs. These simplified theories are valid under several conditions related with the physical parameters of the DOE. In Jing et al. ${ }^{3}$ a full analysis of the accuracy of both methods is detailed with the Fourier Modal Method (FMM) as reference. Although the FMM is classified inside the group of rigorous electromagnetic vector theories, the accuracy of this method depends on the number of spatial harmonics used to represent the periodic electromagnetic field. In addition, it is known that the convergence of FMM, as the Rigorous Coupled Wave Theory (RCWT), is high for TE polarization, but it exhibits poor convergence for TM polarization. The Finite-Difference Time Domain (FDTD) method is considered here as the reference instead of FMM. FDTD also belongs to the rigorous EM theories, and it is frequently applied to yield exact predictions of DOEs. However, FDTD has been considered difficult to

Optical Modelling and Design II, edited by Frank Wyrowski, John T. Sheridan, Jani Tervo, Youri Meuret, Proc. of SPIE Vol. 8429, 84291U · (C) 2012 SPIE · CCC code: 0277-786X/12/\$18 · doi: 10.1117/12.922332 
use and it is computationally intensive. Because of that, in this work a parallel FDTD approach has been considered, which has been also accelerated by Graphic Processing Units (GPUs), avoiding the disadvantage of the time costs related with the finite difference methods. Moreover, the application of this numerical method gives us the opportunity to analyze higher orders and manufacturing deficiencies in the DOE.

As mentioned above, the comparison between different theories applied to binary phase gratings and triangular gratings is shown. The shape of the electromagnetic field is also included for both structures by means of the FDTD method. This method is considered here as reference because of its potential in obtaining all the information of the electromagnetic fields and also the possibilities of simulating with the same performance different polarizations and spatial structures.

\section{THEORY}

\subsection{Scalar Diffraction Theory}

In Fig. $1 \Lambda \mathrm{y} h$ represent the period and groove depth, respectively, and $n_{0}$ and $n_{g}$ are the refractive indices of the incident medium and the grating, respectively. The light wave propagates from air $\left(n_{0}=1.00\right)$ through the surface into the substrate material $\left(n_{g}=1.50\right)$.

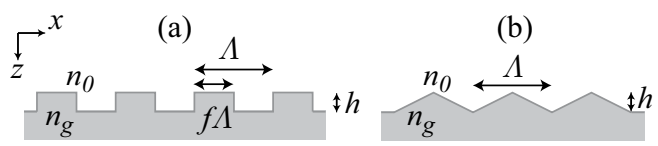

Figure 1. (a) Schematic diagram of diffraction phase grating. (b) Schematic diagram of diffraction triangular grating.

The scalar diffraction efficiency of the SDT can be calculated by using the scalar Kirchhoff diffraction theory, which neglects the vectorial and polarized nature of light. This theory provides reasonably accurate results when the periodicity of the surface profile is much larger than the wavelength of the incident light ${ }^{3}$. Specifically, when $\Lambda / \lambda \geq 20$, physical optics according to Fresnel and Snell's laws can explain the behavior of the diffraction efficiency of these optical elements ${ }^{3,4}$. This simplified theory is based on the general equation for diffraction efficiency $\eta$ in scalar approximation for a periodic structure as ${ }^{3,5}$

$$
\eta(\lambda)=\left|\frac{1}{\Lambda} \int_{0}^{\Lambda} t(x) \exp (-2 j m x / \Lambda) d x\right|^{2},
$$

where $t(x)$ is a function defined as the ratio of transmitted (or reflected) and incident wave amplitudes at location $x$, and $m$ is the diffracted order. Taking into account (1) and the geometry of the problem fully detailed in Fig 1a, the diffraction efficiencies for the zero and first order can be easily derived ${ }^{3,5}$

$$
\begin{gathered}
\eta_{0}=\left(n_{g} \cos \theta_{g} / n_{0} \cos \theta_{0}\right) \tau^{2}\left(\theta_{0}\right)[1-2 f(1-f)(1-\cos \Delta \varphi)], \\
\eta_{m \neq 0}=\left(n_{g} \cos \theta_{g} / n_{0} \cos \theta_{0}\right) \tau^{2}\left(\theta_{0}\right)\left[\left(1 / m^{2} \pi^{2}\right)(1-\cos (2 m \pi f))(1-\cos \Delta \varphi)\right],
\end{gathered}
$$

whith $\theta_{0}$ being the angle of incidence. The rays inside the grating have an angle respect to the $x$ axes $\theta_{g}$ that can be obtained via Snell's Law. The Fresnel transmission coefficient is included by means of $\tau\left(\theta_{0}\right)$, and $\Delta \varphi=2 \pi h / \lambda\left(n_{g} \cos \theta_{g}\right.$ $n_{0} \cos \theta_{0}$ ) is the phase difference between two parallel rays incident on the grating at an angle $\theta_{0}$.

Regarding triangular gratings, the diffraction efficiency for the zero and first order for normal incidence is

$$
\begin{aligned}
& \eta_{0}=\sin ^{2}\left(\pi h\left(n_{g}-n_{0}\right) / \lambda\right) /\left(\pi h\left(n_{g}-n_{0}\right) / \lambda\right)^{2}, \\
& \eta_{ \pm 1}=\left(\frac{2 \pi h\left(n_{g}-n_{0}\right) \cos \left(\pi h\left(n_{g}-n_{0}\right) / 2 \lambda\right)}{\pi h\left(n_{g}-n_{0}\right)-\lambda \pi^{2}}\right)^{2} .
\end{aligned}
$$

\subsection{Effective Medium Theory}

The EMT takes a subwavelength grating as an anisotropic optical thin film with effective refractive indices. These indices are obtained from series expansions of transcendental functions, in terms of $\Lambda / \lambda^{6}$. Here, the zeroth-order and the 
second-order EMT are used to predict the diffraction efficiencies compared with the results calculated by the RCWT and the FDTD.

$$
\begin{gathered}
n_{T E}^{(2)}=\left[\left(n_{T E}^{(0)}\right)^{2}+\frac{1}{3}\left(\frac{\Lambda}{\lambda}\right)^{2} \pi^{2} f^{2}(1-f)^{2}\left(n_{g}^{2}-n_{0}^{2}\right)^{2}\right]^{1 / 2}, \\
n_{T E}^{(0)}=\left[(1-f) n_{0}^{2}+f n_{g}^{2}\right]^{1 / 2} .
\end{gathered}
$$

To apply the EMT, the diffraction gratings shown in Fig. 1 can be approximated by a large number of lamellar layers, modeling the new structure as a stack of homogenous thin films. Each layer of the stack is defined by a characteristic matrix that can be combined with the $N$ layers of the assembly for building the characteristic matrix of the system.

$$
\left[\begin{array}{l}
B \\
C
\end{array}\right]=\left\{\prod_{q=1}^{N}\left[\begin{array}{cc}
\cos \delta_{q} & j \sin \delta_{q} / \eta_{q} \\
j \eta_{q} \sin \delta_{q} & \cos \delta_{q}
\end{array}\right]\right\}\left[\begin{array}{l}
1 \\
\eta_{g}
\end{array}\right],
$$

where $\delta_{q}=2 \pi h n(q)_{T E} \cos \theta_{q} / N \lambda$ is the phase of the $q$ th layer, $n(q)_{T E}$ represents the effective indices of refraction of the $q$ th layer and $\eta_{q}=\eta_{0} n(q)_{T E} \cos \theta_{q}$ for TE polarization, $\eta_{0}$ is the optical admittance in free space $\left(\eta_{0}=\left(\varepsilon_{0} \mu_{0}\right)^{1 / 2}\right)$, and $\eta_{s}$ is the optical admittance in the substrate material. As detailed above, the angles $\theta_{q}$ can be calculated form Snell's Law.

The vector diffraction theory described can also be applied to surface relief gratings. The triangular surface relief grating can be decomposed into planar gratings ${ }^{4,7}$ as shown in Fig. 2. Each layer has an effective refractive index that can be obtained by means of (6)-(7).

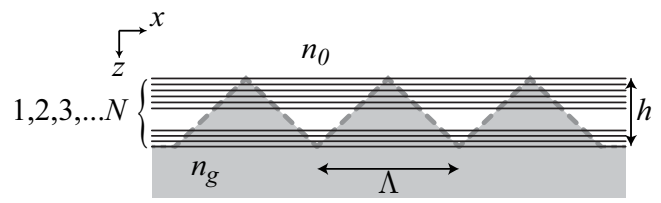

Figure 2. Planar gratings resulting from the decomposition of the surface-relief grating into $N$ thin gratings.

\subsection{Rigorous Coupled Wave Theory}

In order to understand how light propagates inside a periodic medium, many numerical methods have been developed, such as the modal theory, first proposed by Wang and $\mathrm{Tamir}^{8-10}$ et al. and applied to holography by Burkhardt ${ }^{11}$ or the

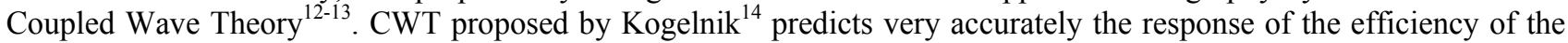
first and second order for volume phase gratings. Nonetheless, the accuracy decreases when more than two orders are present in the grating. The Rigorous Coupled Wave Theory doesn't disregard second derivatives in the CW equations and allow more than two orders. The RCW introduced by Moharam and Gaylord ${ }^{15-17}$ has accomplished the task of explaining a great number of physical situations associated with diffraction gratings of different kinds. Since the RCW is well known only a brief description is given here.

We will study the propagation of light inside binary phase gratings and triangular gratings. In all cases the conductivity inside the grating is supposed to be zero and the relative permittivity in the hologram is expressed as:

$$
\begin{gathered}
\varepsilon_{0}=(1-f) n_{0}^{2}+f n_{g}^{2}, \\
\varepsilon_{p}=\left(n_{g}^{2}-n_{0}^{2}\right) \frac{\sin (\pi p f)}{\pi p},
\end{gathered}
$$

with $f$ being the duty cycle of the square pattern illustrated in Fig. 1 and $p$ the index of the Fourier component of the permittivity. In all cases it has been assumed a value of $f=1 / 2$. Fig 3 shows the pattern of binary and triangular gratings as a function of the number of harmonics considered in the Fourier series. As can be seen in Fig 3, a fewer number of harmonics reproduces better the profile of the triangular grating than that of the binary grating that needs more harmonics for an accurate reproduction of the boundaries of the grating. 
(a)

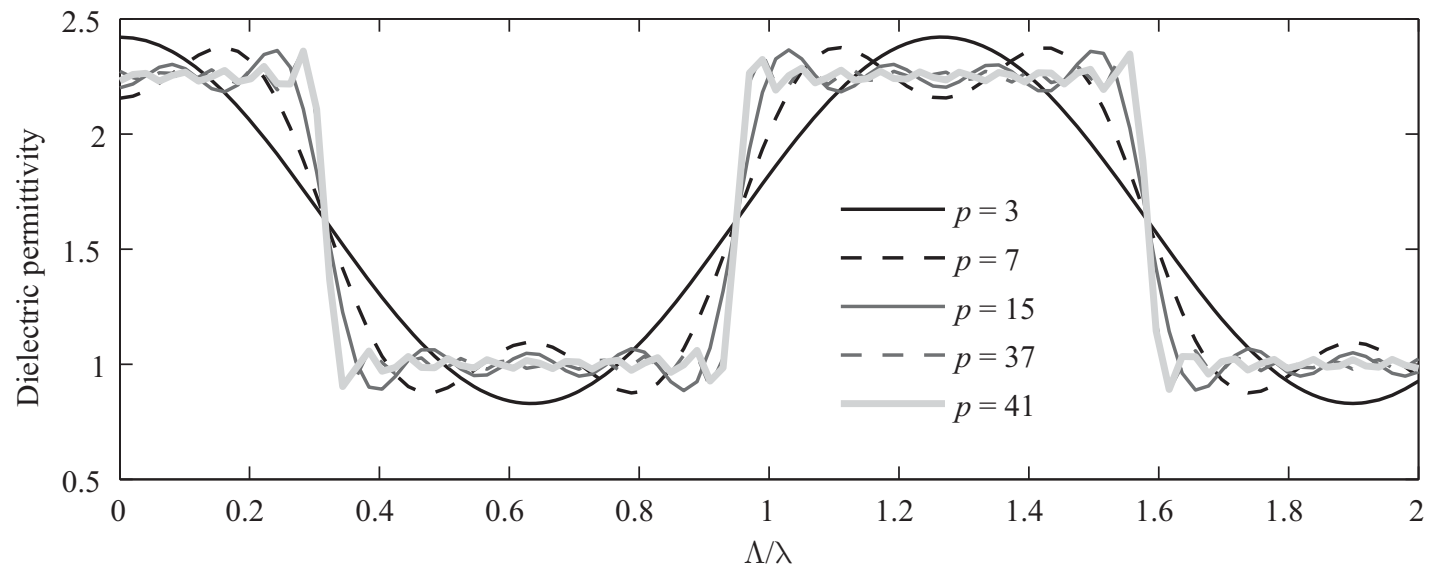

(b)

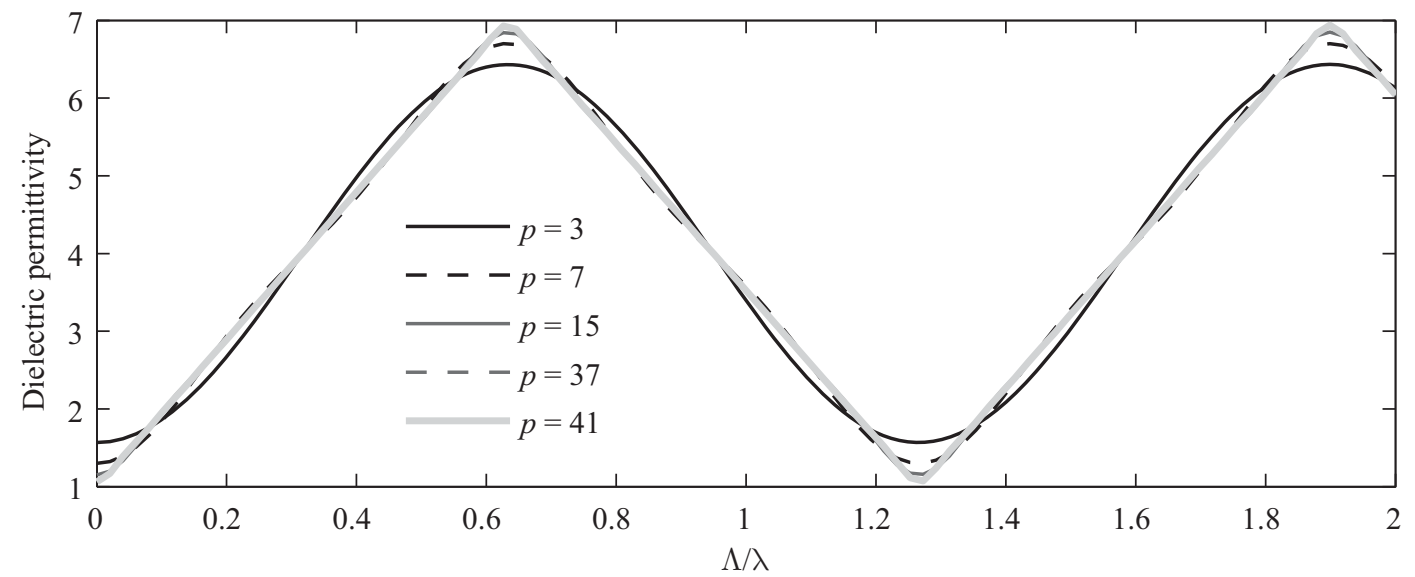

Figure 3. Dielectric permittivity for binary (a) and triangular (b) gratings as a function of the number of harmonics considered in the Fourier series.

Following the $\mathrm{CW}$ and RCW approaches the electric field inside the hologram is supposed to be an infinite sum of orders in the form:

$$
\begin{gathered}
E_{y}=\sum_{i} S_{y i}(z) \exp \left(-j k_{x i} x\right) \\
H_{x}=-\sum_{i}\left(\frac{\varepsilon_{0}}{\mu_{0}}\right)^{1 / 2} U_{y i}(z) \exp \left(-j k_{x i} x\right),
\end{gathered}
$$

where $S_{y i}$ and $U_{x i}$ are the amplitude of the $i$ th diffracted order and $k_{x i}$ is the propagation vector defined by the Floquet theorem

$$
\left.k_{x i}=k_{0}\left[n_{0} \sin \theta_{0}-i(\lambda / \Lambda)\right]\right] .
$$

Taking into account the Maxwell's curl equations for TE polarization and Eq. (9)-(10) a $2 N$ system of equations can be established in matrix form as follows

$$
\left[\begin{array}{l}
\dot{\mathbf{S}}_{y} \\
\dot{\mathbf{H}}_{x}
\end{array}\right]=\left[\begin{array}{cc}
\mathbf{0} & \mathbf{I} \\
\mathbf{A} & \mathbf{0}
\end{array}\right]\left[\begin{array}{l}
\mathbf{S}_{y} \\
\mathbf{H}_{x}
\end{array}\right],
$$


where the upper dot represents the derivative with respect to variable $z$. The matrix $\mathbf{A}$ is related with the propagation vector of each diffraction order considered (13) and with the Fourier series of the permittivity (9-10). The band structure of this matrix and the boundary conditions applied are fully detailed in the work of Neipp et al ${ }^{18}$.

The solution of the amplitude of the different orders can be obtained in terms of the eigenvalues and eigenvector of the matrix $\mathbf{A}$ imposing the adequate boundary conditions ${ }^{18}$.

\subsection{Finite Difference Time Domain Method}

In 1966, Yee proposed a numerical method of solving Maxwell's equations by continuously stepping forward in time in discrete steps and solving a series of linear equations ${ }^{19-20}$. The FDTD technique is a robust analysis tool applicable to a wide variety of complex problems. Specifically, it is useful when nonlinearities exist and transient analysis is required. Nonetheless, the analysis of the diffraction pattern of diffractive optical elements is interesting because of the potential of the method in visualize the electromagnetic field pattern at different time steps and also the possibility of consider different polarizations and spatial schemes without too much effort. The range of physical phenomena which a FDTD algorithm can emulate is a result of how precisely Maxwell's equations are implemented. The equations here considered are the following Maxwell's time-dependent equations:

$$
\begin{gathered}
\frac{\partial \tilde{\mathbf{D}}}{\partial t}=\frac{1}{\sqrt{\varepsilon_{0} \mu_{0}}} \nabla \times \mathbf{H}, \\
\tilde{\mathbf{D}}(\omega)=\varepsilon_{r}(\omega) \tilde{\mathbf{E}}(\omega) \\
\frac{\partial \mathbf{H}}{\partial t}=-\frac{1}{\sqrt{\varepsilon_{0} \mu_{0}}} \nabla \times \tilde{\mathbf{E}},
\end{gathered}
$$

where $\varepsilon_{0}$ is the electrical permittivity in farads per meter, $\varepsilon_{r}$ is the medium's relative complex permittivity constant, that has been assumed real, $\mu_{0}$ is the magnetic permeability in henrys per meter. The flux density is denoted by $\tilde{\mathbf{D}}$ and both, $\tilde{\mathbf{D}}$ and $\tilde{\mathbf{E}}$ are normalized respect to the vacuum impedance $\eta_{0}=\left(\mu_{0} / \varepsilon_{0}\right)^{1 / 2}$.

The FDTD algorithm used here is based on the $\mathrm{Yee}^{19}$ lattice. The electric field components $\mathbf{E}$ and the magnetic field components $\mathbf{H}$ are centered in a bi-dimensional cell so that every $\mathbf{E}$ component is surrounded by four circulating $\mathrm{H}$ components, and every $\mathbf{H}$ component is surrounded by four circulating $\mathbf{E}$ components ${ }^{21-23}$. As a result, the Maxwell's curl equations can be discretized and solved by using the central-difference expressions, for both the time and space derivatives. Theses equations can be easily derived from the Maxwell's equations, and are fully detailed in the works of Sullivan et $\mathrm{al}^{21}$, Frances et $\mathrm{al}^{23}$ and $\mathrm{Oh} \mathrm{et} \mathrm{al}^{24}$, for instance. In order to simulate unbounded free space a simplified version of the Perfectly Matched Layers (PML) developed by Berenger ${ }^{25-27}$ has been implemented and related with the illumination method, a Total Field/Scattered Field ${ }^{21-22,28}$ formulation has been also considered.

Regarding the FDTD implementation in the GPU it must be said that, electromagnetic field update is performed by several CUDA (Compute Unified Device Architecture) cores focused on solving each field component. This architecture provides de possibility of invoking a large number of threads in order to execute operations over a big amount of data. These threads are arranged into blocks of $256 \times 2$, and the number of blocks is established by the resources of the GPU. The difference between modern multi-core CPUs and GPUs architecture is illustrated in Fig. 4. A CUDA program is initialized by the CPU in the host and all data to be processed is transmitted via bus PCI-Express into the GPU. The next step is to execute the application inside the GPU and download the results from the GPU back to the host memory. 


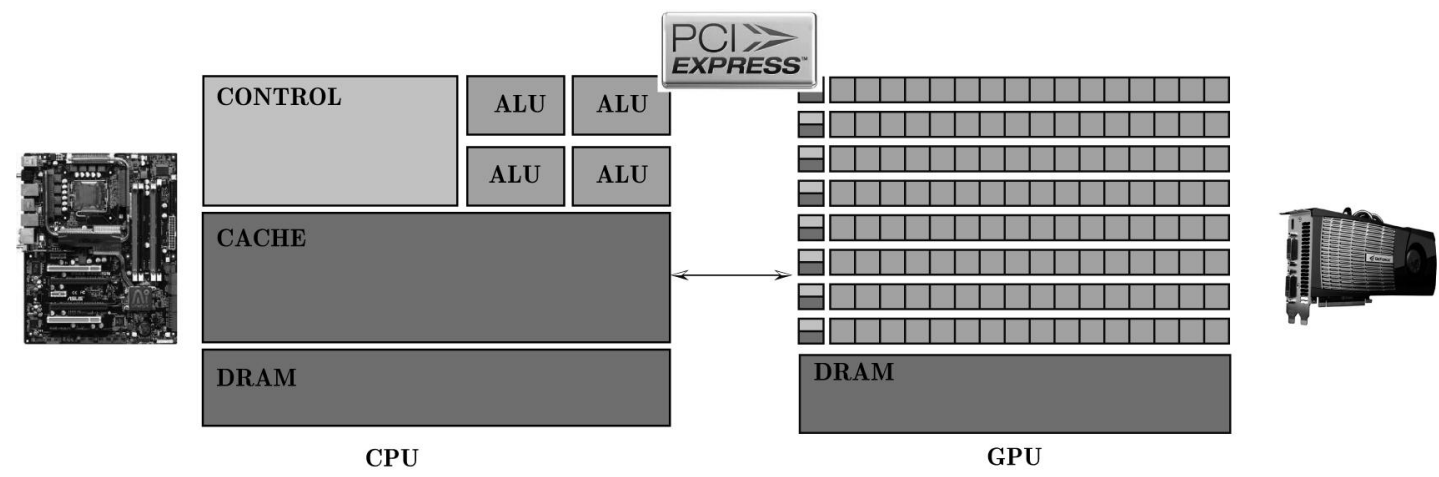

Figure 4. Comparison between CPU and GPU architectures.

Table 1 shows the processing time of the FDTD method accelerated with GPU and with basic CPU processing. The ratio of improvement achieved shows a great impact in time processing as the simulation size increases being in the worst case near of 20 times faster than the basic CPU version.

Table 1: Time simulation of the sequential version of the FDTD method compared with the GPU implementation.

\begin{tabular}{c|ccc} 
MCells & $\mathrm{T}_{\mathrm{CPU}}(\mathrm{s})$ & $\mathrm{T}_{\mathrm{GPU}}(\mathrm{s})$ & $\mathrm{T}_{\mathrm{GPU}} / \mathrm{T}_{\mathrm{GPU}}$ \\
\hline \hline 0.452 & 2.900 & 0.096 & 30.12 \\
1.721 & 18.752 & 0.070 & 26.971 \\
3.810 & 55.154 & 2.154 & 25.610 \\
6.720 & 121.084 & 5.240 & 23.112 \\
10.446 & 231.212 & 10.081 & 22.955 \\
\hline
\end{tabular}

\section{RESULTS}

\subsection{Binary phase gratings}

The results related with the analysis carried out with the different theories described are summarized in Fig 5. In all cases only TE polarization has been considered. The comparison between the diffraction efficiencies obtained with SDT, RCWA and FDTD methods as a function of the $h / \lambda$ parameter are shown in Fig $5 \mathrm{a}, 5 \mathrm{c}$ and $5 \mathrm{e}$, which corresponds with different normalized grating periods: 2, 5 and 8 respectively. As can be seen in these graphs, the RCWA and FDTD methods provide similar results in all cases. However, the accuracy of the scalar treatment degrades as the depth increases. More specifically, the validity of the zero order SDT prediction improves along the normalized depth $(h / \lambda)$ for greater values of $\Lambda / \lambda$. The vector calculations for the \pm 1 order are quite close to the scalar approximation for $\Lambda / \lambda=5, \Lambda / \lambda$ $=8$ and for $\Lambda / \lambda=2$ when the normalized depth is less than 0.5 .

Fig $4 \mathrm{~b}$ shows the comparison of vectorial theories for $\Lambda / \lambda=1$ and Bragg angle of incidence also for the zero and first orders. The degree of similarity between both curves is significant and in this case the SDT has been not included due to its poor behaviour for this value of the $\Lambda / \lambda$ parameter.

The relative error of the RCWA theory as a function of the number of harmonics in the Fourier series of the grating profile is represented in Fig 4e. The error is calculated referred to the solution obtained with 41 harmonics and it is represented as a function of the normalized depth for $\Lambda / \lambda=2$ at normal incidence. As can be seen, the results obtained with 15 harmonics could be considered valid, since the degree of improvement compared with 7 harmonics is considerable. 
\begin{tabular}{l}
$\begin{array}{l}\text { OFDTD (0th) } \\
\times \text { FDTD }( \pm 1 \text { th })\end{array}-$ Scalar (0th) - Scalar (0th) - RCWA (0th) \\
\hline
\end{tabular}

(a)

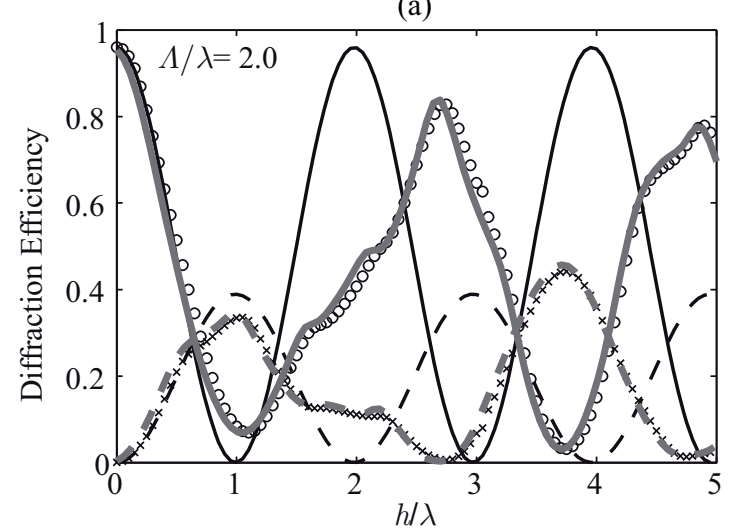

(c)

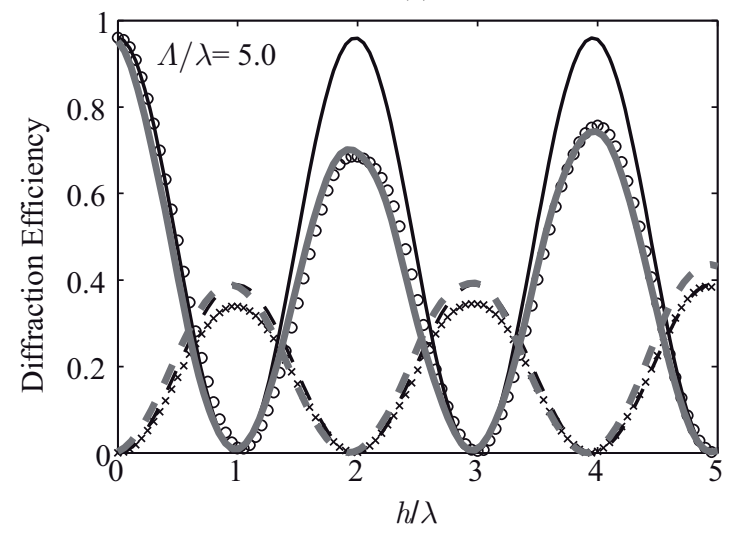

(e)

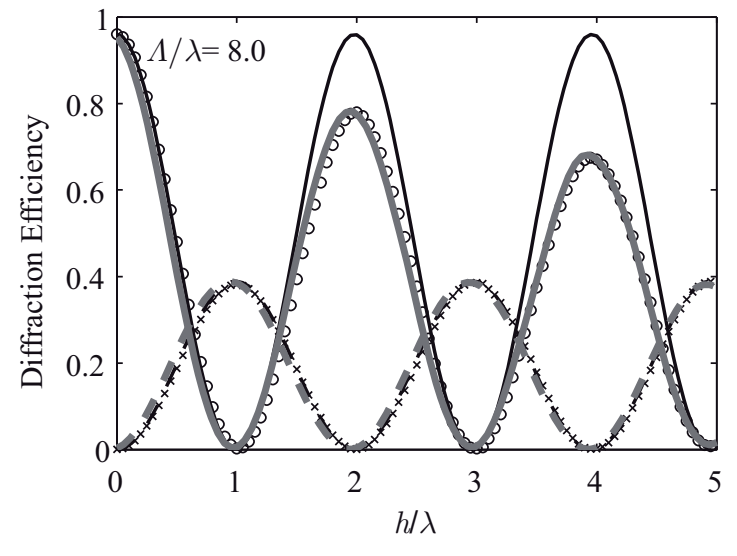

o FDTD (0th) RCWA (0th)

$\times \operatorname{FDTD}( \pm 1$ th $)--\operatorname{RCWA}(0$ th $)$

(b)

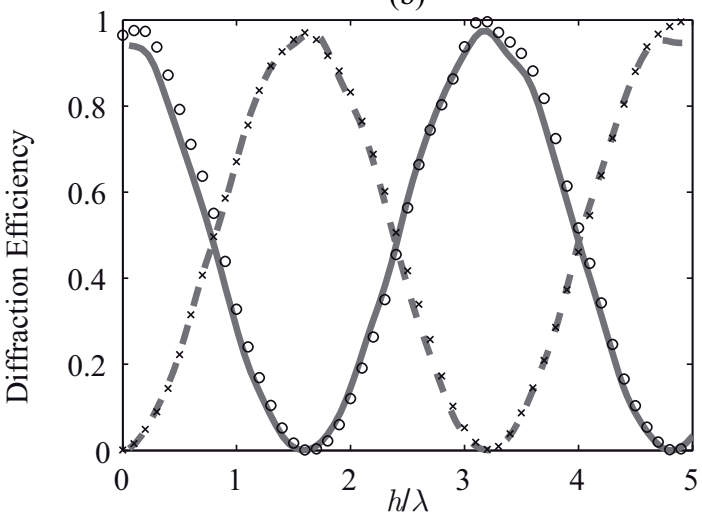

(d)

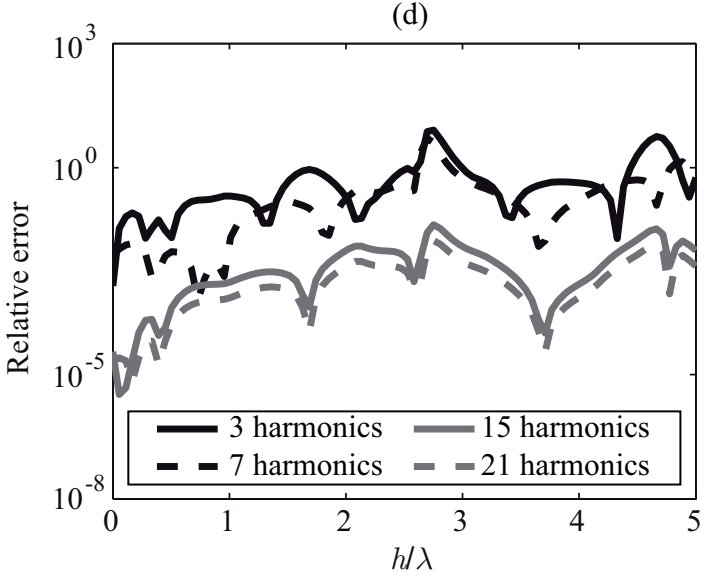

(f)

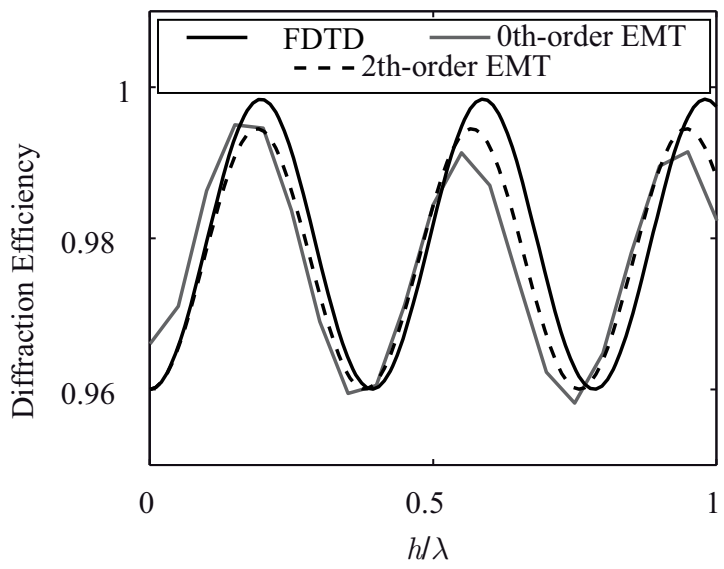

Figure 5. Results for binary phase gratings as a function of the normalized depth. (a), (c) and (d) shows the comparison between SDT, FDTD and RCWA of the diffraction efficiency for different values of $\Lambda / \lambda$. (b) Analysis of the RCWA and FDTD results for $\Lambda / \lambda=1$ and Bragg angle of incidence. (d) Relative error as a function of the number of harmonics considered in the Fourier series. (f) Comparison between EMT and FDTD. In all cases the FDTD parameters are the following: $2048 \times 2048$ cells, $\lambda_{0}=633 \mathrm{~nm}, \Delta=\lambda_{0} / 40$ and $\Delta t=\Delta\left(\varepsilon_{0} \mu_{0}\right)^{1 / 2} / 2$. 
(a)

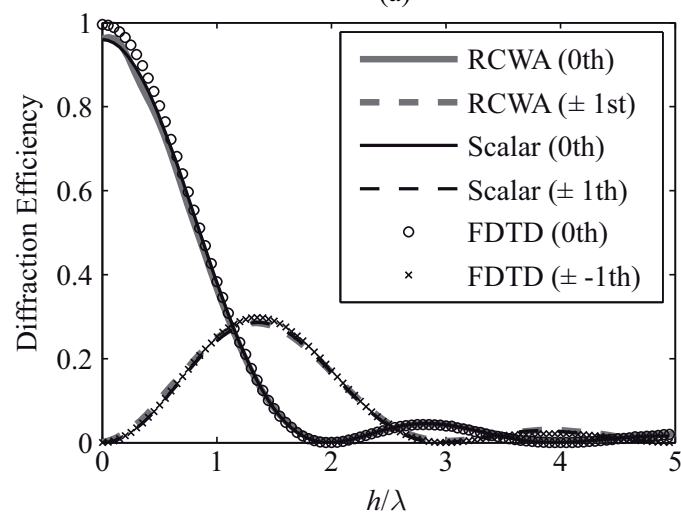

(c)

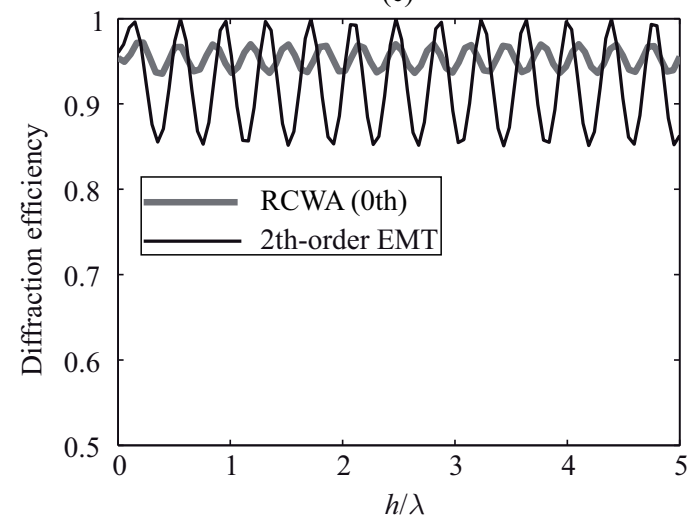

(b) \begin{tabular}{ll|}
$\circ \operatorname{FDTD}(0 \mathrm{th})$ & $\mathrm{RCWA}(0 \mathrm{th})$ \\
$\times \operatorname{FDTD}( \pm 1 \mathrm{st})--$ & $\mathrm{RCWA}( \pm 1 \mathrm{st})$
\end{tabular}

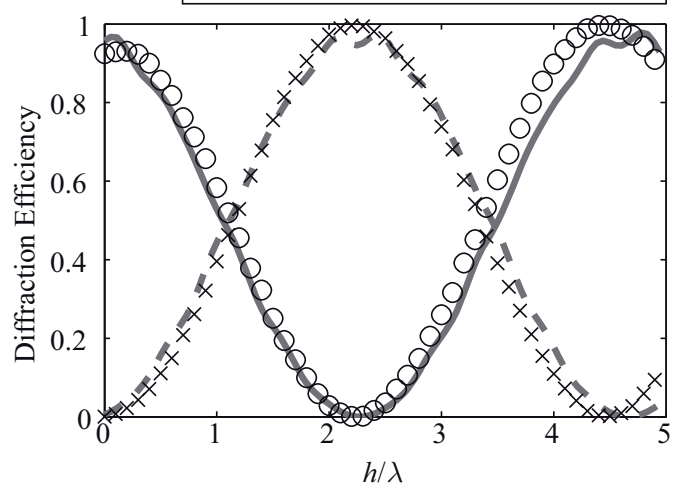

(d)

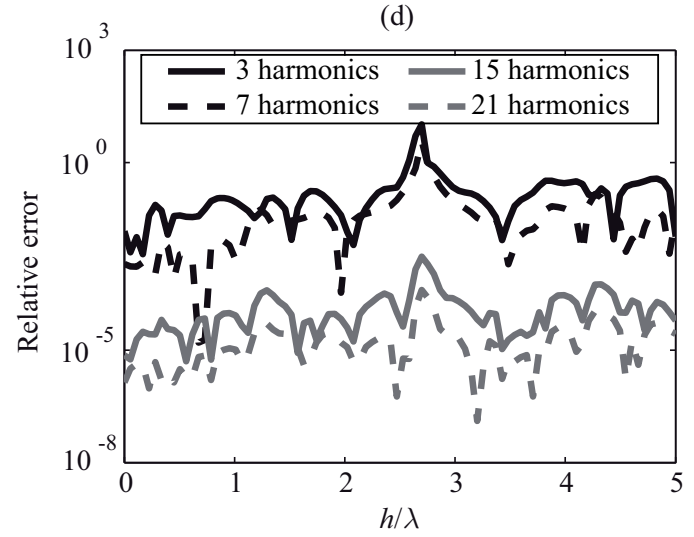

(e)

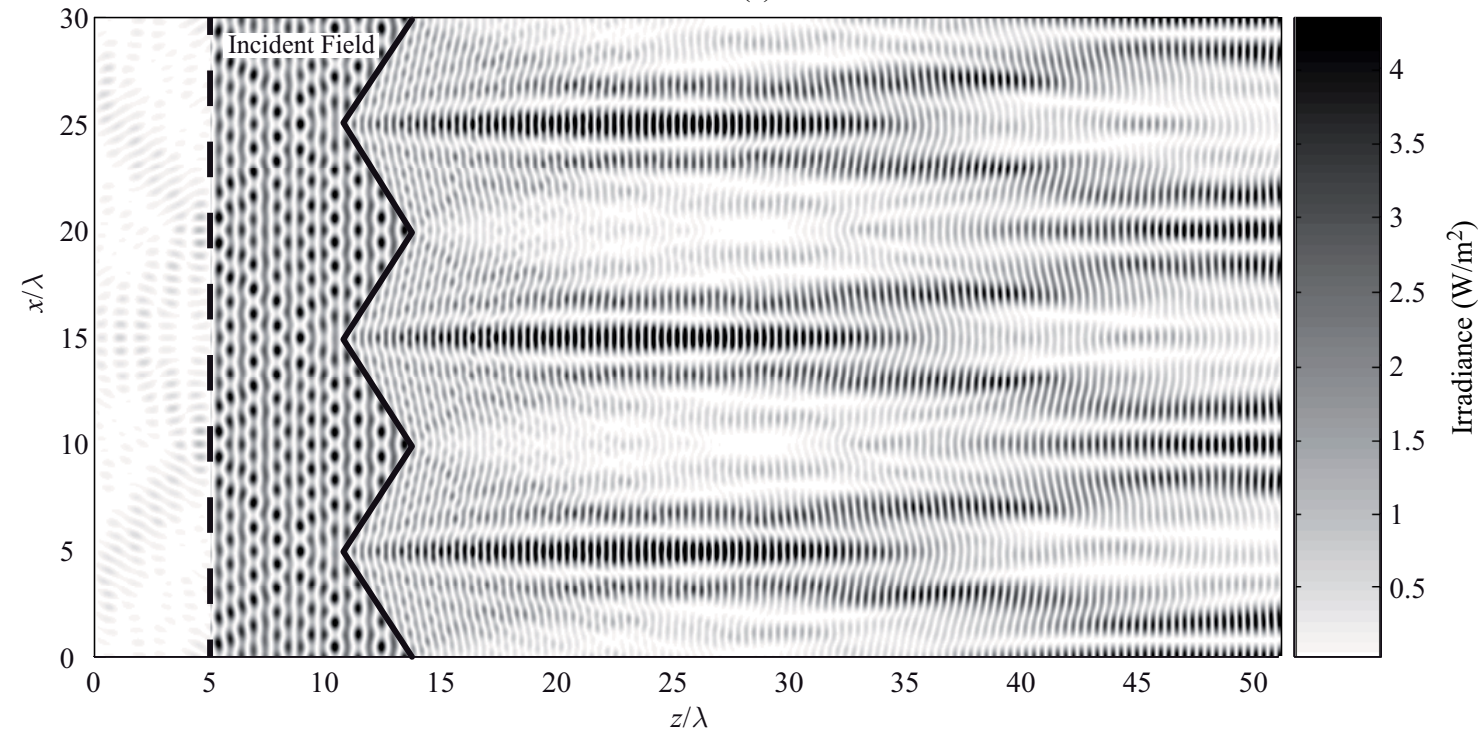

Figure 6. Results for triangular gratings as a function of the normalized depth. (a) Comparison between SDT, FDTD and RCWA of the diffraction efficiency for $\Lambda / \lambda=10$. (b) Analysis of the RCWA and FDTD results for $\Lambda / \lambda=1$ and Bragg

angle of incidence. (c) Comparison between EMT and FDTD. (d) Relative error as a function of the number of harmonics considered in the Fourier series. (f). Distribution of the irradiance as a function of the space for a triangular grating with $\Lambda / \lambda=10$ and $h / \lambda=3$. In all cases the FDTD parameters are the following: $2048 \times 2048$ cells, $\lambda_{0}=633 \mathrm{~nm}, \Delta$ $=\lambda_{0} / 40$ and $\Delta t=\Delta\left(\varepsilon_{0} \mu_{0}\right)^{1 / 2} / 2$. 
Regarding EMT theory, the results for high spatial frequency gratings with EMT are compared with the FDTD method. Specifically, the results shows a good agreement as a function of $h / \lambda$ for $\Lambda / \lambda=0.6$ and both versions of the EMT, $0^{\text {th }}$ order and $2^{\text {nd }}$ order respectively. When the period of the diffraction phase grating is much smaller than the wavelength of the incident light, only the zero order diffraction wave exists, and the EMT is valid to estimate the diffraction efficiency.

\subsection{Triangular gratings}

The results related with the analysis of triangular gratings are represented in Fig. 6. In this case, Fig. 6a represents the diffraction efficiency obtained via scalar approximation, RCWA and FDTD for the zero and first order as a function of the normalized depth for $\Lambda / \lambda=10$. The results of these theories agree in great manner due to the large $\Lambda / \lambda$ here considered. As presented below, the diffraction efficiency obtained by means of the RCWA and the FDTD method for $\Lambda / \lambda=1$ are shown in Fig 6b. A good agreement between these theories is also achieved in this case. Fig 6c illustrate the degree of similarity between the results obtained with the RCWA and EMT theory also for $\Lambda / \lambda=0.6$. In this case the results are not as good as those obtained in section 3.1. This disagreement between these theories can be related with the discretization of the triangular profile with subwavelength layers.

Fig $6 \mathrm{~d}$ shows the relative error of the RCWA prediction for the specific case $\Lambda / \lambda=1$ as a function of the normalized depth and for different number of harmonics. As in Fig. 5d the error is reduced as the number of harmonics becomes greater. However, for triangular profiles the error obtained by the same number of harmonics is lower than for the binary profile. This trend is also corroborated with the similarities of the profiles in Fig 3, where it can be identified a better approach with less harmonics for the triangular grating shown in Fig. $3 b$.

In addition, it has been included in Fig 6e a distribution of the square of the electric field component $\left(E_{z}\right)$, since this magnitude is proportional to the irradiance. This capture corresponds to a simulation with the parameters listed in Fig. 4 caption with $\Lambda / \lambda=10$. The time step simulation selected ensures that the steady-state has been reached. This illustration summarizes the potential of the FDTD method and its capability of obtaining much more information than the diffraction efficiency parameter. Polarization effects, such as the tunneling light phenomena can be easily identified with this method.

\section{CONCLUSIONS}

The results obtained with different vectorial theories and scalar approximations applied to analyze binary phase gratins and triangular gratings are presented. The results show the accuracy of the formalisms and their range of applicability. Some results obtained by Jing et $\mathrm{al}^{3}$ have been corroborated and other theories such as RCWA and FDTD method have been included. Moreover, triangular profiles have been also analyzed achieving good results between the theories implemented. For the specific case of RCWA, the number of harmonics considered in the Fourier series of the grating profile has been analyzed identifying that the triangular profile is less restrictive and can be easily modeled with less harmonics than the binary phase grating. The potential of the FDTD theory with GPU computing provides quite good results compared with the RCWA analysis providing much more information and more flexibility for modeling different DOEs.

Acknowledgements: This work was supported by the "Ministerio de Economía y Competitividad" of Spain under projects FIS2011-29803-C02-01 and FIS2011-29803-C02-02 and by the "Generalitat Valenciana" of Spain under project PROMETEO/2011/021.

\section{REFERENCES}

[1] Moharam, M. G. and Gaylord, T. K., "Diffraction analysis of dielectric surface-relief gratings", Journal of the Optical Society of America, 72(10), 1385-1392 (1982).

[2] Petit, R, ed., [Electromagnetic Theory of Gratings], Springer-Verlag, Berlin, (1980).

[3] Jing, X. and Jin, Y., "Transmittance analysisi of diffraction phase grating", Applied Optics, 50(9), C11-C17 (2011).

[4] Hoshino, T. Banerjee, S. Itoh, M. and Yatagai, T., "Diffraction pattern of triangular grating in the resonance domain", Journal of the Optical Society of America A, 26(3), 715-722(2009).

[5] Cowan, J., "Aztec surface-relief volume diffractive structure", Journal of the Optical Society of America A, 7(8), 1529-1544 (1990).

Proc. of SPIE Vol. 8429 84291U-9 
[6] Rytov, S. M., "Electromagnetic properties of a finely stratified medium", Sov. Phys. JETP, 2, 466-475 (1956).

[7] Moharam, M. G. and Gaylord, T. K., "Three-dimensional vector coupled-wave analysis of planar grating diffraction", Journal of the Optical Society of America, 73, 1105-1112 (1983).

[8] Tamir, T., Wang, H. C. and Oliner, A. A.," Wave propagation in sinusoidally stratified dielectric media," IEEE Transactions on Microwave Theory. MTTT-12, 232-335 (1964).

[9] Tamir, T. and Wang, H. C, "Scattering of electromagnetic waves by a sinusoidally stratified half-space: I. Formal solution and analysis approximations," Canadian Journal of Physics, 44, 2073-2094 (1966).

[10] T. Tamir, "Scattering of electromagnetic waves by a sinusoidally stratified half-space: II. Diffraction aspects of the Rayleigh and Bragg wavelengths," Canadian Journal of Physics, 44, 2461-2494 (1966).

[11] Burckhardt, C. B. "Diffraction of a plane wave at a sinusoidally stratified dielectric grating," Journal of the Optical Society of America, 56, 1502-1509 (1966).

[12] Solymar, L. and Cooke, D. J., [Volume Holography and Volume Gratings], Academic, London, p 79, (1981).

[13] Syms, R. R. A., [Practical Volume Holography], Clarendon Press, Oxford, (1990).

[14] Kogelnik, H., "Coupled Wave Theory for Thick Hologram Gratings," The Bell System Technical Journal, 48(9), 2909-2947 (1969).

[15] Moharam, M. G. and Gaylord, T. K., "Rigorous Coupled-Wave Analysis of planar-grating diffraction," Journal of Optical Society of America, 71(7), 811-818 (1981).

[16] Moharam, M. G. and Gaylord, T. K., "Rigorous Coupled-Wave analysis of grating diffraction -E-Mode polarization and lossles," Journal of Optical Society of America, 73(4), 451-455 (1983).

[17] Moharam, M. G., Grann, E. B., Pommet, D. A. and Gaylord, T. K., "Formulation for stable and efficient implementation of the rigorous coupled-wave analysis of binary gratings," Journal of Optical Society of America A: Optics and Image Science, and Vision, 12(5), 1068-1076 (1995).

[18] Neipp, C, Álvarez, M. L., Gallego, S., Ortuño, M., Sheridan, J. D., Pascual, I. and Beléndez, A., “Angular responses of the first diffracted order in over-modulated volume diffraction gratings," Journal of Modern Optics, 51(8), 1149-1162 (2004).

[19] Yee, K. S., "Numerical solution of initial boundary value problems involving Mawell's equations in isotropic media," IEEE Trans. Antennas Propag. 14, 302-307 (1966).

[20] Miskiewicz, M. N., Bowen, P. T. and Escuti, M. J., "Efficient 3D FDTD analysis of arbitrary birefringent and dichroic media with obliquely incident sources," Proc. SPIE 8255, 82550W-1-10 (2012).

[21] Sullivan, D. M., [Electromagnetic simulation using the FDTD method], IEEE Press Editorial Board, (2000).

[22] Taflove, A., [Computational electrodynamics: The Finite-Difference Time-Domain Method], Artech House Publishers, (1995).

[23] Francés, J., Pérez-Molina, M., Neipp, C., Beléndez, A., "Rigorous interference and diffraction analysis of diffractive optic elements," Comput. Phys. Commun. 182(12), 1963-1973 (2010).

[24] Oh, C. and Escuti, M. "Time-domain analysis of periodic anisotropic meda at oblique incidence: an efficient FDTD implementation, "Optics Express, 14(24), 11870-11884 (2006).

[25] Berenger, J. P., "A perfectly matched layer for the absorption of electromagnetic waves," J. Comput. Phys., 114(2), 185-204 (1994).

[26] Berenger, J. P., "Three-dimensional perfectly matched layer for the absorption of electromagnetic waves," J. Comput Phys., 127(2), 363-379 (1996).

[27] Sullivan, D. M., "A simplified PML for use with the FDTD method," IEEE Microwave and Guided Wave letters 6(2), 97-99 (1996).

[28] Jiang, Y.-N., Ge D-B., Ding, S. J., "Analysis of TF-SF boundary for 2D-FDTD with plane p-wave propagation in layered dispersive and lossy media," Progr. Electromagn. Res. 83(12), 157-172 (2008). 